\title{
RELATIVISTIC MINIMAL SURFACES
}

\author{
Jens HOPPE and Hermann NICOLAI \\ Institut für Theoretische Physik, Universität Karlsruhe, PO Box 6380, D-7500 Karlsruhe, Fed. Rep. Germany
}

Received 26 June 1987

\begin{abstract}
We find classical solutions to the equations of motion of an $M$-dimensional surface moving in a higher-dimensional embedding space-time for arbitrary $M$. In the case of closed membranes, solutions exist for any topological type (genus).
\end{abstract}

Whereas in string theories one has a fairly complete knowledge of most classical and quantum mechanical aspects, much less is known about higher dimensional objects. Even classical solutions are scarce and a full quantum mechanical analysis appears very difficult with present technology. Several years ago, in an attempt to develop a quantum theory of relativistic surfaces, J. Goldstone and one of the authors (J.H.) were able to relate the (mass) ${ }^{2}$ spectrum of a closed surface to the $N \rightarrow \infty$ limit of an $\mathrm{SU}(N)$ invariant large- $N$ matrix model, but no substantial progress in this direction has been made since. A new impulse to studying membranes was given by Kikkawa and Yamasaki [1] who were the first to exhibit rigidly rotating flat membranes as classical solutions. As these membranes stabilize themselves by rotating in two independent planes, they require the space-time dimensions to be at least five, which might explain that they were not found before the revival of Kaluza-Klein theories.

In this letter, we present a new class of solutions to the classical equations of motion of a closed surface (of arbitrary dimension) which correspond to pulsating and/or rigidly rotating objects minimally embedded into euclidean spheres. The space-time dimensions for these solutions to exist have to be at least $2 M+3$, if $M$ is the dimension of the surface. For closed membranes we observe that such solutions exist for all topological types, i.e. arbitrary genus $g$ of the 2-surface ( $g=0$ requiring $D \geqslant 7, D \geqslant 9$ for all higher $g$ ). In addition, we generalize the solution for a flat, open 2-surface found in ref. [1] to any dimensionality $M$.
One motivation for presenting our results is the fact, that semi-classical calculations for closed membranes are now possible. Also we hope that new light will be shed on the results obtained in ref. [2].

The classical equations of motion of an $M$-dimensional extended object whose action $S$ is proportional to the invariant volume of the $(M+1)$ dimensional surface $\psi$ that is swept out in Minkowski space are

$$
\begin{aligned}
& \partial_{\alpha}\left(\sqrt{G} G^{\alpha \beta} \partial_{\beta} x^{\mu}\right)=0, \\
& \quad \mu=0,1, \ldots, D-1, \quad \alpha, \beta=0, \ldots, M,
\end{aligned}
$$

where $G^{\alpha \beta}$ and $G$ are, respectively, the inverse and $(-)^{M}$ times the determinant of the metric tensor $G_{\alpha \beta}=\partial_{\alpha} x^{\mu} \partial_{\beta} x_{\mu}$ induced on $\psi ; x^{\mu}=x^{\mu}\left(\sigma^{0}, \ldots, \sigma^{M}\right)$ describes $\psi$, and $\partial_{\alpha}$ denotes differentiation with respect to the parameter $\sigma^{\alpha}$; our space-time metric convention is $(+-\ldots-)$.

In order to have a physical picture of $\psi$, we choose $\sigma^{0}$ to be the time coordinate $t$ of some Lorentz frame $F$. Then

$x^{\mu}=(t, \boldsymbol{x}(t, \Omega))$,

where $\Omega$ stands for $\left(\sigma^{1}, \ldots, \sigma^{M}\right)$, and the $(D-1)$ dimensional vector $\boldsymbol{x}(t, \Omega)$ now describes the timedependent shape, as seen in $F$, of the $M$-dimensional surface whose dynamics we are interested in. Then

$$
\begin{gathered}
G_{\alpha \beta}=\left(\begin{array}{cc}
1-x^{2} & -x \partial_{r} x \\
-\boldsymbol{x} \partial_{r} \boldsymbol{x} & -\partial_{r} \boldsymbol{x} \partial_{s} \boldsymbol{x}
\end{array}\right), \\
r, s=1, \ldots, M, \quad \dot{\boldsymbol{x}}=\partial \boldsymbol{x} / \partial t
\end{gathered}
$$


and the action reads

$S=-\kappa \int_{t_{1}}^{t_{2}} \mathrm{~d} t \int \mathrm{d} \Omega \sqrt{G}$,

with $\kappa$ a parameter of dimension energy/(length $)^{M}$ $x$ (the surface tension).

The equations of motion (1) represent a set of coupled, nonlinear, partial differential equations. When looking for solutions, one has two realistic chances:

- to make technically simplifying assumptions, and/or

- to use physical intuition of what some solutions might look like.

While the latter could lead one directly to the ansatz

$\boldsymbol{x}(t, \Omega)=x(t) D(t) \boldsymbol{m}(\Omega)$,

corresponding to the simple motions of pulsation (described by a scalar function $x(t)$ ) and rotation (described by a time-dependent real orthogonal matrix $D(t))$ ), the above ansatz also comes out naturally when one follows the first path:

An obvious simplification is to use the reparametrisation invariance of the theory to choose $\Omega$ such that

$G_{0 r}=-\dot{\boldsymbol{x}} \partial_{r} \boldsymbol{x}=0$.

The equations of motion then become

$\partial_{0}\left(\sqrt{g} / \sqrt{1-\dot{x}^{2}}\right)=0$,

and, already using (7),

$$
\begin{aligned}
& \ddot{\boldsymbol{x}}+\frac{1}{2} \partial_{r}\left(\dot{\boldsymbol{x}}^{2}\right) g^{r s} \partial_{s} \boldsymbol{x} \\
& \quad-\left(1-\dot{\boldsymbol{x}}^{2}\right)(1 / \sqrt{g}) \partial_{r}\left(\sqrt{g} g^{r s} \partial_{s} \boldsymbol{x}\right)=0,
\end{aligned}
$$

where $g$ and $g^{r s}$ are the determinant and inverse, respectively, of

$g_{r s}=\partial_{r} x \cdot \partial_{s} x$.

(7) will play a crucial role throughout; in particular, it tells one that there exist infinitely many constants of motion $\sqrt{G} G^{00}=\mu(\Omega)$, and thus reflects the timeindependent reparametrisation invariance of $(8)$, and its scale invariance $(a \boldsymbol{x}(a t, \Omega)$ solves (8) if $\boldsymbol{x}(t, \Omega)$ does).

A second essential simplification occurs whenever $g_{r s}(t, \Omega)$ factorizes into a product $\tilde{g}(t) \bar{g}_{r s}(\Omega)$. But this immediately leads to (5); in addition, it practically requires, via (7), $\dot{\boldsymbol{x}}^{2}$ to be independent of either time or $\Omega$, which again simplifies (8). For open surfaces $\dot{\boldsymbol{x}}^{2}$ has to be 1 on the boundary and thus (excluding singular solutions, i.e. $1-\dot{x}^{2}=0$ everywhere) only the first possibility can be realized. For closed surfaces, both possibilities seem to exist in principle, although the solutions described below are all of the second kind.

Before discussing these closed-surface solutions (which will be our main interest), let us mention the generalisation of the Kikkawa-Yamasaki solution for a rotating flat, open 2-surface to arbitrary $M$ :

$\boldsymbol{x}(t, \Omega)=\left(f_{1}(\Omega) \boldsymbol{n}_{1}(t), \ldots, f_{M}(\Omega) \boldsymbol{n}_{M}(t), 0, \ldots, 0\right)$,

where $\boldsymbol{n}_{r}(t)=\left(\cos \omega_{r} t, \sin \omega_{r} t\right)$ and the $f_{r}(\Omega)$ are arbitrary functions, apart from having to satisfy $\sum_{r=1}^{M} \omega_{r}^{2} f_{r}^{2}(\Omega)=1$ on the boundary of the open surface. Let us look at how they manage to satisfy (8) (for $M=2$, there is but little need to organize the cancellation, but for higher $M$ it is both necessary and instructive). With

$g_{r s}(t, \Omega)=\partial_{r} f \cdot \partial_{s} f=\widehat{g}_{r s}(\Omega)$,

$\boldsymbol{r}$ denoting $\left(f_{1}, \ldots, f_{M}\right),(8)$ reads

$$
\begin{aligned}
& -\omega_{\alpha}^{2} f_{\alpha}+\left(\sum_{1}^{M} \omega_{\beta}^{2} f_{\beta} \partial_{r} f_{\beta}\right) \bar{g}^{r s} \partial_{s} f_{\alpha} \\
& -\left(1-\sum_{1}^{M} \omega_{\beta}^{2} f_{\beta}^{2}\right) \frac{1}{\sqrt{\bar{g}}} \partial_{r}\left(\sqrt{\bar{g}} \bar{g}^{r s} \partial_{s} f_{\alpha}\right)=0 .
\end{aligned}
$$

The second term is equal to $\omega_{\alpha}^{2} f_{\alpha}$, and the third term is identically 0 , because of the identities

$\partial_{r} f_{\beta} \bar{g}^{r s} \partial_{s} f_{\alpha}=\delta_{\alpha \beta}$,

and

$(1 / \sqrt{\bar{g}}) \partial_{r}\left(\sqrt{\bar{g}} \bar{g}^{r s} \partial_{s} f_{\alpha}\right)=0 \quad(\alpha, \beta=1, \ldots, M)$,

which are trivially valid in the gauge $f_{r}=\sigma^{r}$ (and hence true generally because of reparametrisation invariance).

For closed surface solutions one cannot have such a simple cancellation, as for (13) and (14) to be true, 
it is crucial that there are exactly as many functions $f_{\alpha}$ as parameters $\sigma^{r}$ and in the same way that a closed string necessarily needs at least two dimensions to live in, a closed $M$-dimensional surface needs at least $M+1$ functions $f_{\alpha}(\Omega)$ to be described at a given time $t$. In fact, in contrast with the above cancellation, our closed-surface solutions will have the second term in (8) identically zero, while the third will be the interesting one, leading to a great variety of solutions. As we now want to give a somewhat deductive description of closed surface solutions that are a mixture of pulsation and rigid rotation, let us go back to the ansatz (5). In order to have

$$
\begin{aligned}
& \dot{\boldsymbol{x}}^{2}(t, \Omega)=\dot{\boldsymbol{x}}^{2}(t) \boldsymbol{m}^{2}(\Omega) \\
& \quad+x^{2}(t) \boldsymbol{m}^{\mathrm{T}}(\Omega) \dot{D}^{\mathrm{T}}(t) \dot{D}(t) \boldsymbol{m}(\Omega),
\end{aligned}
$$

independent of $\Omega$, we first of all choose $m(\Omega)$ to be a unit vector:

$m^{2}(\Omega)=1$.

Then $\boldsymbol{x}(t, \Omega)$ has the simple interpretation of a rotating $M$-dimensional surface embedded in a sphere $\mathrm{S}^{D-2}$ of time-dependent radius $x(t)$. To get a feeling for this, one may think of a closed string gliding on top of a pulsating sphere $S^{2} \subset \mathbb{R}^{3}$. Although we will see that already in this case one needs at least two independent (commuting) rotations (thus the necessity to go out of $\mathbb{R}^{3}$, to at least $\mathbb{R}^{4}$, i.e. $D=5$ ) this picture of the rotating closed string will be helpful throughout. To make the second term in (15) independent of $\Omega$, we choose

$D(t)=\exp [A \varphi(t)]$

$A^{2} \boldsymbol{m}(\Omega)=-\mathbf{1} \cdot \boldsymbol{m}(\Omega)$.

Thus

$\boldsymbol{x}(t, \Omega)=x(t)[\cos \varphi(t) 1+\sin \varphi(t) A] \boldsymbol{m}(\Omega)$.

Each point of the surface is being rotated in the timeindependent plane spanned by $\boldsymbol{m}(\Omega)$ and $A \boldsymbol{m}(\Omega)$; alternatively, $D(t)$ can be thought of as a product of mutually commuting rotations around the same angle $\varphi(t)$.

To have $G_{0 r}=0$, one has to require

$\left(\partial_{r} \boldsymbol{m}\right)(A \boldsymbol{m})=0$,

which we satisfy by choosing $\boldsymbol{m}=\left(n_{1}, n_{2}, \ldots, n_{\mathrm{d}}, 0, \ldots, 0\right), \quad d \leqslant(D-1) / 2$

and

$A_{i j}=\left(\begin{array}{rrr}0 & -\mathbf{1} & 0 \\ \mathbf{1} & 0 & 0 \\ 0 & 0 & 0\end{array}\right)$,

where 1 is the $d \times d$ unit matrix. Putting together (19), (21) and (22), we thus look for solutions of the form

$\boldsymbol{x}(t, \Omega)=x(t)(\cos \varphi(t) \boldsymbol{n}, \sin \varphi(t) \boldsymbol{n}, 0, \ldots, 0)$,

where $\left.\boldsymbol{n}(\Omega)=n_{1}, \ldots, n_{d}\right)$ is a $d$-dimensional unit vector describing an embedding of an $M$-dimensional closed surface in $\mathrm{S}^{d-1}$.

The $\mu=0$ part of the equations of motion (compare (7))

$\partial_{0}\left\{[x(t)]^{M} \sqrt{\bar{g}(\Omega)} / \sqrt{1-\dot{x}^{2}}\right\}=0$

tells one that

$\dot{x}^{2}+x^{2} \dot{\varphi}^{2}+(x / C)^{2 M}=1$

- $C$ a constant with the dimension of a length - which allows one to bring the equation for $x$ into the form

$\ddot{x}-x^{2 M-2} / C^{2 M} \nabla^{2} \boldsymbol{x}=0$,

where

$\nabla^{2}=(1 / \sqrt{\bar{g}}) \partial_{r} \sqrt{\bar{g}} \bar{g}^{r s} \partial_{s}, \quad \bar{g}_{r s}=\partial_{r} \boldsymbol{n} \cdot \partial_{s} \boldsymbol{n}$,

is the covariant Laplacian on the $M$-surface swept out by $\boldsymbol{n}(\Omega)$. Calculating $\ddot{\boldsymbol{x}}$, one finds

$x^{2}(t) \dot{\varphi}(t)=L=$ const.,

corresponding to the conservation of angular momentum $J$; in fact, $J$ is proportional to $L$ with a factor which depends on the shape of the surface described by $\boldsymbol{n}(\Omega)$. From (25), it follows that

$\ddot{x}-L^{2} / x^{3}+M x^{2 M-1} / C^{2 M}=0$,

which is compatible with (26) provided that $n(\Omega)$ is an eigenfunction of the Laplace operator (27) with eigenvalue $-M$, i.e.

$\nabla^{2} \boldsymbol{n}(\Omega)=-M \boldsymbol{n}(\Omega)$.

But this is precisely the requirement that $\boldsymbol{n}$ describes 
a minimal surface in $S^{d-1}$. Namely the variation of $\int \mathrm{d} \Omega \sqrt{\bar{g}}$, together with the constraint $n^{2}=1$, i.e.

$\delta\left(\int \mathrm{d} \Omega\left[\sqrt{\bar{g}}+\lambda(\Omega)\left(\boldsymbol{n}^{2}-1\right)\right]\right)=0$

yields

$\nabla^{2} \boldsymbol{n}=[2 \lambda(\Omega) / \sqrt{\bar{g}}] \boldsymbol{n}$,

and, from $\boldsymbol{n}^{2}=1$,

$$
\begin{aligned}
0 & =\boldsymbol{n} \cdot \partial_{r} \boldsymbol{n}=\partial_{s}\left(\sqrt{\bar{g}} \bar{g}^{s r} \boldsymbol{n} \cdot \partial_{r} \boldsymbol{n}\right) \\
& =\sqrt{\bar{g}}\left(M+\boldsymbol{n} \cdot \nabla^{2} \boldsymbol{n}\right) .
\end{aligned}
$$

Combining (32) and (33), one gets (30).

While minimal surfaces and geodetic curves have been studied since the early days of analysis, the subject of minimal embeddings of higher dimensional closed surfaces in euclidean spheres is comparatively new. Still, there exist some useful results. E.g., closed two-dimensional minimal surfaces in $\mathrm{S}^{3}$ have been found for any genus [3]. However, explicit parametrisations are only known for $S^{2}$ and $S^{1} \times S^{1}$; they read

$\boldsymbol{n}=(\sin \theta \cos \varphi, \sin \theta \sin \varphi, \cos \theta)$

$$
\begin{gathered}
\theta \in[0, \pi], \varphi \in[0,2 \pi] \quad \text { for } S^{2}, \\
\boldsymbol{n}^{\prime}=(\cos \theta, \sin \theta, \cos \varphi, \sin \varphi) \\
\theta \in[0,2 \pi], \varphi \in[0,2 \pi] \text { for } S^{1} \times S^{1} .
\end{gathered}
$$

For certain surfaces with self-intersections explicit parametrisations are kown for all $g$ [3].

Minimal 2-surfaces (mainly $\mathrm{S}^{2}$ ) in $\mathrm{S}^{n>3}$ have been studied in refs. [4,5]. For higher dimensional surfaces it is known, that (probably all) higher spheres $\mathrm{S}^{n>3}$ admit infinitely many distinct (non-congruent) embeddings of $\mathrm{S}^{n-1}$ in contrast with $n=2$ and $n=3$, where there is only one [6].

The minimality condition becomes rather suggestive when one thinks of the example of a closed string: "minimal" curves on $\mathrm{S}^{2}$ are the equators, and it is intuitively clear that they are the ones that can balance the string tension by rotating around their "center of gravity"; any other curve would be "pushed outwards" and simultaneously shrink to 0 . On the other hand, one must be aware that our three-dimensional intuition can easily go wrong in higher dimensions; e.g., Karcher, Pinkall and Sterling [7] recently found a two-dimensional closed minimal surface that does not divide $S^{3}$ into two parts of equal volume.

Let us return to the equations for $x(t)$ and $\varphi(t)$, namely (25), (28) and (29), which can be viewed as combining the time dependence of the pulsation and rotation, respectively, to the problem of a classical particle (of unit mass) moving in two dimensions, with the rotationally invariant, anharmonic potential

$V(x)=\frac{1}{2}(x / C)^{2 M}$.

Integrating (29) gives

$\frac{1}{2} \dot{x}^{2}+\frac{1}{2} L^{2} / x^{2}+V(x)=$ const. $=E$,

corresponding to the conservation of "energy". However, $E$ is not arbitrary, but fixed to be $1 / 2$, according to (25). A simple (and stable) solution of $(25),(28)$ and $(29)$ is given by

$x(t)=R=$ const.,$\quad \varphi(t)=\omega t$,

$R^{2} \omega^{2}=M /(M+1)$,

which corresponds to the particle moving on a circle of radius $R$ with constant angular velocity $\omega=\sqrt{M /(M+1)} / R$. Note that (38) explicitly requires $\dot{\varphi}$ to be different from 0 , and that (going back to the original picture) the last equation in (38) tells one that all points on the surface move with the velocity $\sqrt{M /(M+1)}$.

Other solutions of (37) are obtained by straightforward integration

$t=\int \frac{\mathrm{d} x}{\sqrt{1-L^{2} / x^{2}-2 V(x)}}$.

They correspond to a simultaneous rotation and pulsation of the $M$-surface. For $L=0$, there is no rotation and the solution becomes singular as the diameter of the surface passes through zero (for the special case of a two-dimensional sphere, this solution was discovered long ago [8]). From (37), it is clear that for fixed energy $\kappa \int \mathrm{d} \mu$, the solution with maximum $L$ is obtained with $\dot{x}=0$ and thus coincides with (38). Quite explicitly, it reads

$$
\begin{aligned}
& \boldsymbol{x}(t, \Omega)=\sqrt{M /(M+1)}(1 / \omega) \\
& \quad \times(\cos \omega t \boldsymbol{n}(\Omega), \sin \omega t \boldsymbol{n}(\Omega), 0, \ldots) .
\end{aligned}
$$

We would like to thank M. Schlichenmayer, H. 
Karcher and U. Pinkall for discussions, in particular for leading us to refs. [3-7]. Also we want to acknowledge discussions with J. Wess, M. Scheunert, M. Scholl and U. Marquard. One of us (J.H.) would like to thank the theory group at Karlsruhe for their warm hospitality as well as atmospheric motivation and financial support, G. Roepstorff for making possible this one month leave from Aachen University, during which the results of this paper have been obtained, J. and T. Wess for both of the above, and U.B., G.B., B.W., and C.H. for their help, and love.

\section{References}

[1] K. Kikkawa and M. Yamasaki, Progr. Theor. Phys. 76 (1986) 1379.
[2] J. Hoppe, Quantum theory of a relativistic surface..., MIT PhD Thesis (1982); and Florence 1986 workshop on Constraints theory and relativistic dynamics (World Scientific, Singapore, Longhi/Lusanna) pp. 267-276.

[3] H.B. Lawson Jr., Ann. Math. 92 (1970) 335.

[4] R.L. Bryant, J. Diff. Geom. 17 (1982) 455.

[5] E. Calabi, J. Diff. Geom. 1 (1967) 111.

[6] W.-Y. Hsiang, Ann. Math. 118 (1983) 61; Invent. Math. 74 (1983) 351.

[7] H. Karcher, U. Pinkall and I. Sterling, New minimal surfaces in $\mathrm{S}^{3}$, preprint Bonn MPI 86-27.

[8] P.A. Collins and R.W. Tucker, Nucl. Phys. B 112 (1976) 150. 